\title{
Resolutions Against Uniqueness $^{\dagger}$
}

\author{
Kenji Lota and Ulf Hlobil
}

\begin{abstract}
The paper presents a new argument for epistemic permissivism. The version of permissivism that we defend is a moderate version that applies only to explicit doxastic attitudes. Drawing on Yalcin's framework for modeling such attitudes, we argue that two fully rational subjects who share all their evidence, prior beliefs, and epistemic standards may still differ in the explicit doxastic attitudes that they adopt. This can happen because two such subjects may be sensitive to different questions. Thus, differing intellectual interests can yield failures of uniqueness. This is not a merely pragmatic phenomenon.
\end{abstract}

\section{Introduction}

Is it possible for two fully rational subjects with the same evidence to have different doxastic attitudes towards the same proposition? Advocates of epistemic uniqueness think that this is not possible (Christensen, 2007; Dogramaci \& Horowitz, 2016; Elga, 2007; Feldman, 2007; Greco \& Hedden, 2016; Horowitz, 2014, 2018, 2019; Levinstein, 2017; Matheson, 2011; Schultheis 2018; Stapleford 2018; White, 2005, 2013). Permissivists disagree (Jackson 2019; Kelly, 2013; Meacham, 2013, 2019; Raleigh, 2017; Rosa, 2012; Rosen, 2001; Schoenfield, 2014, 2018; Simpson 2017; Thorstad, 2019; Titelbaum \& Kopec, 2019). In this paper, we offer a new argument for

\footnotetext{
${ }^{\dagger}$ This is a post-peer-review, pre-copyedit version of an article published in Erkenntnis. The final authenticated version is available online at: http://dx.doi.org/10.1007/s10670-021-00391-z. Please cite published version.
} 
permissivism and, hence, against uniqueness. More precisely we will argue for a so-called interpersonal and moderate version of permissivism about explicit beliefs. The arguments here are not restricted to qualitative attitudes, but apply to quantitative attitudes as well. We will clarify what this means in due course.

By way of foreshadowing, here is the intuitive idea behind our argument: Which explicit beliefs you should adopt as a result of receiving new evidence is, in part, a matter of which questions you want to answer, i.e. what is of interest to you (cf. Friedman, 2018). If, e.g., you are looking for your keys and I am looking for my wallet and both are lying on the table and we are both looking at the table, then it is rational for me to form the belief that my wallet is on the table and it is rational for you to form the belief that your keys are on the table. Not forming these beliefs respectively would be less than perfectly rational. If I am not interested in where your keys are, however, it is not irrational for me not to form the explicit belief that your keys are on the table. I may have evidence regarding whether these are keys and who owns them, but I am under no rational obligation to ask myself these questions and to form an explicit belief that these are your keys. Below, we present a way of modeling this phenomenon by taking explicit beliefs to be sensitive to questions, or partitions of modal space (Yalcin, 2018).

Advocates of uniqueness may hold that what is at issue in such cases is a merely pragmatic matter of what evidence we should gather, or they may hold that fully rational agents form explicit beliefs in all the propositions that their evidence supports. We will address such worries in Sections 3 and 4.

In what follows, we first set the stage by identifying our target, sketching relevant aspects of the current debate, and introducing our way of modeling explicit beliefs (Section 2). We then present our argument for permissivism (Section 3). Next, we consider some objections (Section 4). Then we conclude (Section 5). 


\section{Setting the Stage}

In this section, we provide the necessary background. We begin with the debate about uniqueness and permissivism. We then turn to the question how we can model doxastic states in an illuminating way. We end the section by considering whether the version of uniqueness about explicit attitudes that is our target is relevant for the ongoing debate about uniqueness.

\subsection{The Permissivism Debate}

Permissivism is the denial of the so-called epistemic uniqueness thesis (or "uniqueness" for short). Since there are different versions of uniqueness, there are also different versions of permissivism. In this subsection, we will pinpoint the version that we want to defend. To this end, we will formulate a version of uniqueness that is immune to many of the objections that have been voiced in the literature. We will then show that this version nevertheless succumbs to our novel criticism.

Let's start with extant formulations of uniqueness. Feldman formulates uniqueness as follows:

[Uniqueness] is the idea that a body of evidence justifies at most one proposition out of a competing set of propositions (e.g., one theory out of a bunch of exclusive alternatives) and it justifies at most one attitude toward any particular proposition. (Feldman, 2007, p. 405)

By contrast, White formulates uniqueness thus:

Given one's total evidence, there is a unique rational doxastic attitude that one can take to any proposition. (White, 2005, p. 455)

As Titelbaum and Kopec (2019) point out, these two formulations are not equivalent. Our target is closer to White's than to Feldman's uniqueness thesis. More precisely, we are interested in a version of uniqueness that denies that it can be rational for one agent to have a particular 
attitude, such as belief or suspension, regarding a proposition, while another agent with the same evidence has another attitude or no attitude towards the same proposition. Following White's (2005, p. 453) terminology (adjusting it mildly), we call this a moderate version of permissivism, which contrasts with strong versions. Strong versions of permissivism require that one of our two subjects believes a proposition while the other disbelieves it, believing its negation. By contrast, it suffices for the truth of our moderate version of uniqueness that the second subject suspends judgment on the matter or has no explicit attitude towards the proposition. ${ }^{1}$

Concurrently, our discussion mainly focuses on qualitative attitudes. We shall also touch on quantitative attitudes later. This distinction can be found in the literature as the distinction between coarse-grained and fine-grained attitudes (Kopec and Titelbaum, 2016), or belief and credal permissivism (Jackson, 2019). However, our arguments bring out that belief states can be analyzed more finely without appeal to credences. Moreover, our version is interpersonal; it says that a certain difference between two people is possible (Kelly, 2013). Thus, as a first pass, our target version of uniqueness says that two fully rational people with the same evidence must have the same attitude, or lack of any attitude, towards every proposition. ${ }^{2}$

In order to make our results and our task more interesting, we now make our version of uniqueness invulnerable to three worries. This will ensure that our argument is independent of such worries. The first worry is that uniqueness is false because rational agents that are confronted with the same evidence may differ with respect to what evidence they gathered in the past or with respect to evidence about their own abilities or other high-order evidence (Matheson, 2011). In order to neutralize such worries, we require that the two agents share all of their evidence, including past and high-order evidence.

A second worry is that uniqueness is false because different rational agents may have differed in their attitudes before they received any evidence. Subjective Bayesians, e.g., hold that there are different permissible ur-priors (Kelly, 2013; Schoenfield, 2014, 2018; Titelbaum \& Kopec, 2019). To neutralize this worry, we will require that our two agents started with the same initial doxastic attitudes or ur-priors. 
A third worry is that uniqueness is false because different rational agents may have different epistemic standards (Schoenfield, 2014). They may, e.g., be more or less averse to the risk of having false beliefs, relative to the prospect of having true beliefs (Horowitz, 2018; Kelly, 2013). Unfortunately, it is not always clear in the literature what different authors mean by "epistemic standards." We will assume here that whatever epistemic standards are, they are different from the resolutions that we will introduce below. With this assumption in place, we can take this third worry off the table by requiring that our two agents have the same epistemic standards.

Finally, besides immunizing our target from these worries, we want to flag that we are interested in permissivism about explicit doxastic attitudes. For the purposes of this paper, we are willing to grant uniqueness for implicit doxastic attitudes. To the best of our knowledge, the distinction between implicit and explicit doxastic attitudes has not been brought up in the debate. Standard examples in the literature, such as differing opinions by jurors in a jury (e.g. Rosen, 2001; cf. White, 2005), seem to concern primarily explicit doxastic attitudes, as the relevant attitudes are expressed overtly by the agents in these examples. We will consider the issue in Section 2.3. But first, we will clarify our distinction between implicit and explicit doxastic attitudes in the next subsection.

Let's sum up what we said so far by formulating the version of uniqueness that will serve as our target:

UNIQ If two fully rational subjects (1) started with the same initial doxastic attitudes, (2) share all their evidence, and (3) use the same epistemic standards, then they must have the same explicit doxastic attitude (or lack thereof) towards any proposition.

The aim of this paper is to show that UNIQ is false. 


\subsection{Resolutions of Doxastic States}

Any discussion of uniqueness must work with some conception or model of the doxastic states of fully rational subjects. In this subsection, we will introduce the model with which we will work, which we lift, with only minor changes, from Seth Yalcin (2018).

Let's start with a standard possible worlds model of doxastic states (in the spirit of Stalnaker, 1984). We take propositions to be sets of possible worlds, intuitively the worlds in which the proposition is true. ${ }^{3}$ The overall doxastic state of an epistemic subject is modeled by the set of possible worlds that are live options (for being actual) for the agent; intuitively these are the worlds that are "left open" by the subject's beliefs. Let's call this set of worlds the "maximal proposition that is implicitly believed" by the subject. For fully rational subjects, these are the worlds that are not ruled out by their evidence. The subject implicitly believes a proposition, $P$, iff $P$ is a superset of the maximal proposition she implicitly believes. She implicitly disbelieves $P$ iff the intersection of $P$ and her maximal proposition is empty. And if neither is the case, then she neither implicitly believes nor implicitly disbelieves $P$.

Perhaps the most significant drawback of this model of doxastic states is that it builds in logical omniscience (Stalnaker, 1991). Every doxastic state is modeled as being closed under logical consequence. ${ }^{4}$ To see this, notice that if a subject's maximal proposition logically entails $P$, then there are no possible worlds in which the agent's maximal proposition is true but $P$ is false. Hence, the proposition $P$ is a superset of the subject's maximal proposition, which means that the subject believes $P$. Since logical truths are logical consequences of every proposition, it follows that all subjects implicitly believe all logical truths, i.e., they are logically omniscient. Some philosophers argue that fully rational agents must believe all logical truths and all logical consequences of their beliefs (Smithies, 2015; Stapleford, 2018). We use the word "implicit" to mark a sense of "believe" for which that may be defensible. There is, however, also another sense of "believe," let's call it "explicit belief," for which logical omniscience is implausible. At least, we are happy to take this commitment on board as one of our premises. 
How can we model explicit beliefs? Here we follow Seth Yalcin (2018). The key element that Yalcin introduces are "resolutions;" these are partitions of modal space into mutually exclusive and jointly exhaustive regions. Such resolutions can be viewed as the semantic interpretants of questions (Hamblin, 1958). The question "Is my wallet on the table?", e.g., can be modelled by a partition of all possible worlds into two cells, namely those worlds in which my wallet is on the table and those worlds in which it isn't. When I ask the question, I want to know which of these two cells contains the actual world. Similarly, the question "In which of these six drawers are my keys?" can be modelled by a partition of all possible worlds into seven cells, namely one cell that contains all the possible worlds in which my keys are in the first drawer, and similarly for the other five drawers, plus one cell where my keys are not in any of the six drawers. ${ }^{5}$

Now, the explicit beliefs of an agent are answers to questions to which the agent is sensitive. They are cases in which the agent is sensitive to certain differences between possibilities and takes a stand on what actuality is like with respect to those differences, i.e., in which cell or collection of cells of a resolution the actual world lies. To model this, we say that a subject explicitly believes a proposition, relative to a given resolution, just in case the proposition is (exactly) a union of some cells of the resolution and that union is a superset of the maximal proposition that the subject believes implicitly. In pictures like Fig. 1, these are all the combinations of cells that "cover" the entire implicit maximal proposition, e.g., the proposition $R$ that is depicted as a red rectangle in Fig. 1. We say that the belief partition of an agent relative to a resolution is the collection of cells such that each cell contains at least one possible world contained in the maximal proposition that the agent believes implicitly. Belief partitions are the minimal collection of cells that "cover" the entire implicit maximal proposition; they are the explicitly believed analogue of the maximal proposition that the agent believes implicitly.

To illustrate, suppose, e.g., that I explicitly believe that I am a mammal but that I have never considered the question whether it is true that either I am a mammal or the moon is made of green cheese. I don't have any explicit belief regarding the latter proposition; my explicit beliefs are thus not closed under logical consequence. Once the question is raised, however, I, 
as it were, partition the space of possibilities that I rule in or out in a new way, namely, I partition the space into those worlds in which I am either a mammal or the moon is made of green cheese and all other possible worlds. Seeing that I am a mammal (as I believe) and that all the possibilities in which I am a mammal are also possibilities in which I am either a mammal or the moon is made of green cheese, I come to believe the latter proposition. In terms of the model, I rule in the first cell of my new partition, throughout which the latter proposition is true, and I rule out the other cell.

To sum up, we model belief states as partial functions from resolutions to the cells of the resolutions that are left open by what the agent believes. The resolutions for which this function is defined are the resolutions (i.e. questions) to which the agent is sensitive. Figure 1 illustrates the notion of resolution, implicit and explicit belief, and the maximal proposition that an agent believes implicitly.

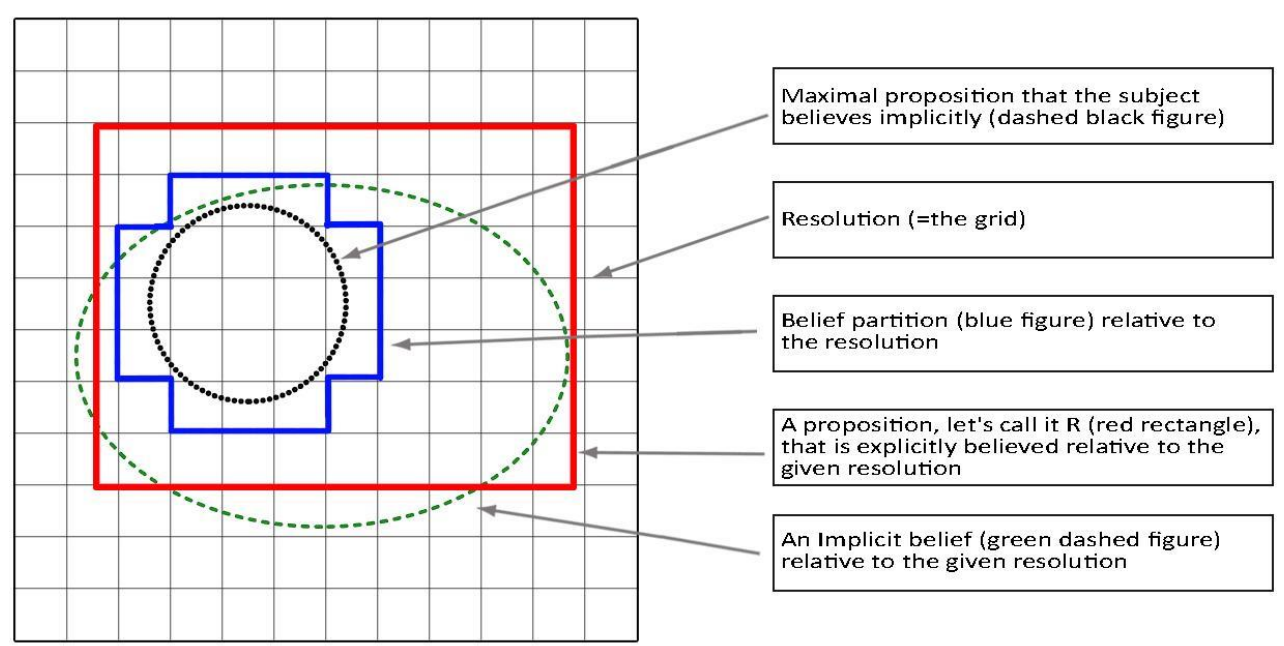

Figure 1: Model of a belief state (in modal space) relative to a resolution; with implicit and explicit beliefs, relative to the depicted resolution (see Fig. 2 of Yalcin $(2018$, p. 32) for a similar illustration and more details).

The idea is that the resolution models the distinctions that the agent makes between possibilities. The worlds in any given cell of the resolution are treated alike by the agent. The agent is not 
distinguishing between these possibilities. If the evidence of an agent leaves it open that one of the worlds in any given cell is the actual world, then the agent should consider the whole cell as a live option for what the world is like.

\subsection{Uniqueness of Explicit or Implicit Attitudes}

Having presented a model of doxastic states, we now revisit how this applies to our topic. A proponent of uniqueness might worry that, properly interpreted, uniqueness is a thesis about implicit and not about explicit attitudes, given the explicit/implicit distinction from the previous section. Before we proceed to our argument for permissivism, we address this worry here. ${ }^{6}$

Before we respond to this worry, two clarifications are in order. Our first clarificatory remark is that we do not claim that the debate about uniqueness should focus exclusively on explicit attitudes. Rather, we hold that the question of uniqueness should be considered for both, explicit and implicit attitudes. For the purposes of this paper, we are happy to accept that uniqueness holds for implicit attitudes, and our question is whether it also holds for explicit attitudes. As a second clarification, we want to stress that our notion of an explicit belief must not be confused with the notion of an occurrent or a conscious belief. Rather, explicit beliefs, in our sense, are beliefs that are (a) not rationally required to be closed under entailment, (b) are integrated with one another only to the extent that they are defined over the same set of questions, and (c) the explicit beliefs that are thus integrated guide our behavior, i.e. they determine how we reason and act. Implicit beliefs, by contrast, are those that are rationally required to be closed under entailment, or not held relative to certain questions, and don't guide behavior and reasoning. Parallel contrasts hold for more fine-grained doxastic attitudes. We will sketch in Section 4 what (a)-(c) amount to in more detail. For now, (a)-(c) shows that we typically have very many explicit beliefs that are unconscious and not occurrent. Until reading this sentence, e.g., your explicit belief that the floor in your house is safe to walk on was probably unconscious. That you nevertheless had that explicit belief, in our terminology, comes out in the 
fact that your actions were guided by the information that the floor in your house is safe to walk on. You were sensitive to the question whether the floor is safe to walk on. With these clarifications out of the way, let's consider the worry that uniqueness should be interpreted as concerning only implicit attitudes.

First, notice that a version of uniqueness that applies only to implicit beliefs concerns only an extreme ideal of rationality. Rational implicit beliefs are closed under entailment, and hence include, e.g., beliefs in all logical and mathematical truths. If considering that rationality implies logical omniscience, then there is only one set of rational beliefs about logic and mathematics. Yalcin's notion of explicit belief is intended to capture a sense in which, e.g., failures of logical omniscience are not failures of rationality. And the kind of irrationality for which a failure to be logically omniscient is sufficient doesn't seem to carry a lot of normative weight (in most contexts). The criticism that one is irrational because one doesn't belief the truth about Goldbach's conjecture, e.g., doesn't strike us as a weighty attack on one's claim to rationality. Insofar as uniqueness is a thesis committed to serious rational criticism, it must target agents' explicit and not one's implicit beliefs. And even if the extremely stringent ideal of rationality that applies to implicit beliefs requires uniqueness, it seems to us that it is an interesting further question whether the same holds for the more realistic notion of rationality that governs explicit beliefs.

Second, the notion of explicit beliefs that we take over from Yalcin is meant to capture the sense in which beliefs guide our behavior, which Yalcin puts by saying that beliefs function as maps that we can use to guide actions. Different inquirers end up with different explicit belief states that allow them to navigate the world differently, which is why we tend to act and reason differently. And our assertions, how we describe the world, what information we share as part of our testimony, and how we think the world should be, vary depending on our explicit beliefs. Even if the attitudes that are governed by ideals of logical omniscience and that don't guide our actions are rationally required to be unique, we think it is an interesting further question whether the doxastic attitudes that guide our actions are also rationally required to be unique. 
Finally, notice that some of the arguments for uniqueness can be understood as arguments for either implicit or explicit beliefs. White (2005; 2013), for example, appeals to the importance of being uniquely rational when deliberating about practical matters, emphasizing actions that yield unfavorable consequences, such as letting a guilty criminal go free or choosing a dangerous path home. Other advocates of uniqueness crucially rely on cases of deference to others' opinions (Greco \& Hedden, 2016; Levinstein, 2017), and testimony (Dogramaci \& Horowitz, 2016). Given our definition of explicit beliefs above, all of these cases plausibly involve explicit and not implicit belief. For the beliefs that figure in practical deliberation, in deference, and in testimony are such that the agents are sensitive to their contents. We do not claim that advocates of uniqueness construe their thesis as clearly about explicit beliefs, in our sense. We merely hold that it is not clear that the debate should concern only implicit attitudes. Hence, our thesis that uniqueness fails for explicit beliefs is relevant to the debate about uniqueness.

\section{An Argument for Permissivism}

In this section, we present our argument for permissivism. The idea behind this argument is that two fully rational subjects who start with the same initial doxastic states, who share all their evidence, and who use the same epistemic standards may nevertheless differ in the questions to which they are sensitive. Hence, these subjects can differ in the resolutions over which their explicit beliefs are defined. Moreover, as we will show, even if their explicit beliefs rule out exactly the same worlds, this may change if both subjects update their doxastic states in light of the same evidence. The upshot is that if we allow for different, equally rational, resolutions, permissivism about explicit beliefs becomes unavoidable. We can formulate our argument in a compressed form as follows:

(I) If two fully rational agents who meet (1)-(3) of UNIQ can be sensitive to different questions, then permissivism about explicit beliefs is true. 
(II) Two fully rational agents who meet (1)-(3) of UNIQ can be sensitive to different questions.

(III) Therefore, permissivism about explicit beliefs is true.

The aim of this section is to defend the premises of this argument. We will defend premise (I) in the next subsection, and we do so within the qualitative framework sketched above. In subsection 3.2, we generalize the result to a quantitative framework. Finally, in subsection 3.3, we will argue that premise (II) is also true.

\subsection{Different Rational Resolutions Imply Permissivism}

Let's take two fully rational agents who started with the same initial doxastic attitudes, who share all their evidence, and who use the same epistemic standards. Moreover, let's suppose that uniqueness is true of implicit beliefs. Hence, our two agents' implicit beliefs rule out exactly the same possible worlds. Can they nevertheless differ in their explicit beliefs? In this subsection, we will show that the answer is "yes."

Take, e.g., the belief state relative to the resolution displayed in Fig. 1. Let's keep the maximal proposition that the agent believes implicitly, and hence all the other implicit beliefs, constant. Let's change merely the resolution. While the red rectangle is a proposition that is explicitly believed relative to the resolution in Fig. 1, it is not explicitly believed relative to the resolution in Fig. 2. 


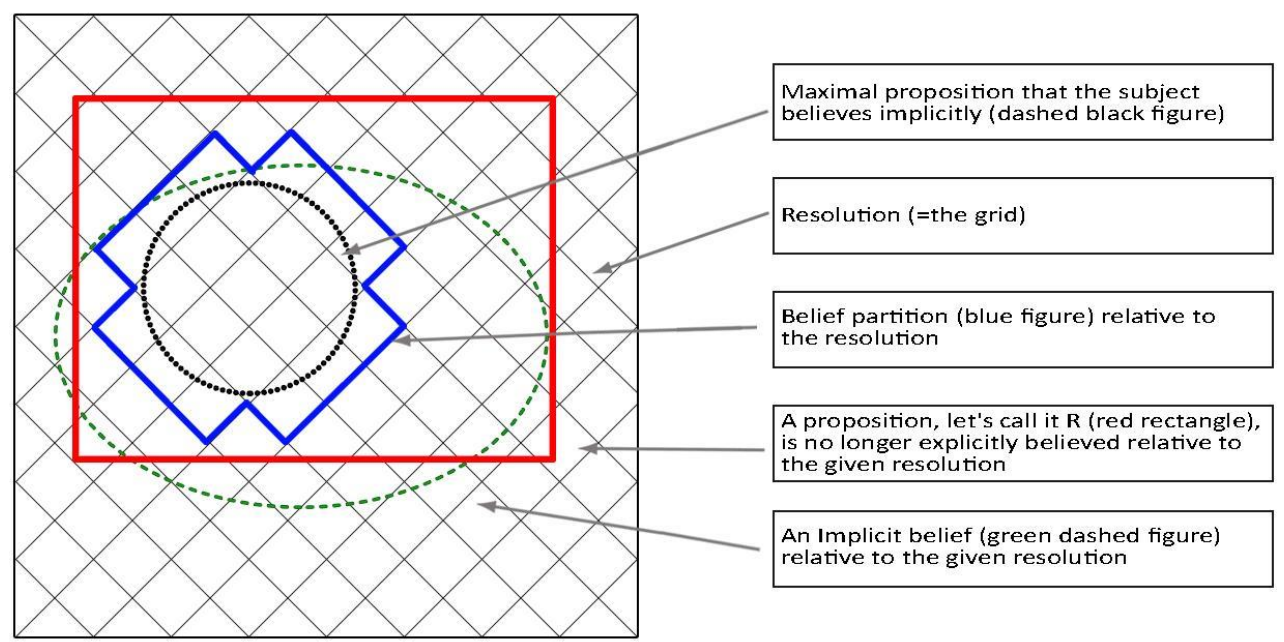

Figure 2: The same belief state as in Fig. 1, except that the resolution has changed. The proposition represented by the red rectangle is no longer explicitly believed.

If one of our subjects is sensitive to the resolution in Fig. 1 and the other subject is not sensitive to this resolution but, e.g., only to the resolution in Fig. 2, then the first subject will explicitly believe the proposition $R$ that is represented by the red rectangle in both figures. The second subject, however, will not explicitly believe $R$. Hence, UNIQ fails in such cases.

Notice that our second subject will not explicitly believe anything that is incompatible with $R$, let alone not- $R$. She will merely fail to believe $R$. According to our view, it is not possible that two rational subjects that meet the conditions (1)-(3) of UNIQ are such that one explicitly believes a proposition and the other explicitly believes the negation of that proposition (or anything entailed by that negation). That is what makes our permissivism moderate.

Let's illustrate our argument by returning to the example with which we started. If you are looking for your keys and I am looking for my wallet and we are both looking at the table, then you will be sensitive to the question whether your keys are on the table, whereas I am not sensitive to that question. As a result, you will have explicit beliefs relative to the resolution that corresponds to the question "Are my keys on the table?", whereas I will not have any explicit beliefs relative to that resolution. In other words, the partial function that models your belief 
state is defined for that resolution, while the partial function that models my belief state is not defined for that resolution. As a result, you explicitly believe that your keys are on the table, while I don't explicitly believe that proposition. Since our story doesn't entail that either of us is less than fully rational, UNIQ fails.

One might think that this result holds only if we allow that our two agents start out with explicit beliefs that rule out different possible worlds. Perhaps, one might think, uniqueness cannot fail if the two agents start with a resolution that cuts exactly along the boundaries of the shared maximal proposition with which they start. Perhaps if we then update the maximal proposition with new evidence by intersecting, this will allow us to hold on to uniqueness. That, however, is not so. To see this, consider the updates on the belief states depicted in Fig. 3.

The two initial belief states in Fig. 3 agree in the possible worlds that are ruled out by the implicit and explicit beliefs of the agents. They differ merely in the resolutions over which they are defined (we assume that they are not defined for the resolutions depicted for the respectively other belief state). Fig. 3 also depicts the results of updating each belief state (relative to the depicted resolution) in light of the same evidence. We can do that by intersecting the maximal proposition that the agents believe implicitly with the incoming evidence to arrive at a new maximal proposition. This is the same for both subjects. However, our two subjects differ in the explicit beliefs that result from this update. We arrive at each subject's new belief partition relative to their respective resolutions in the following way: we include in that partition exactly those cells of their respective partitions that contain at least one world that is contained in the new maximal proposition that both agents believe implicitly. As Fig. 3 shows, this leads to different belief partitions. Hence, UNIQ fails. 

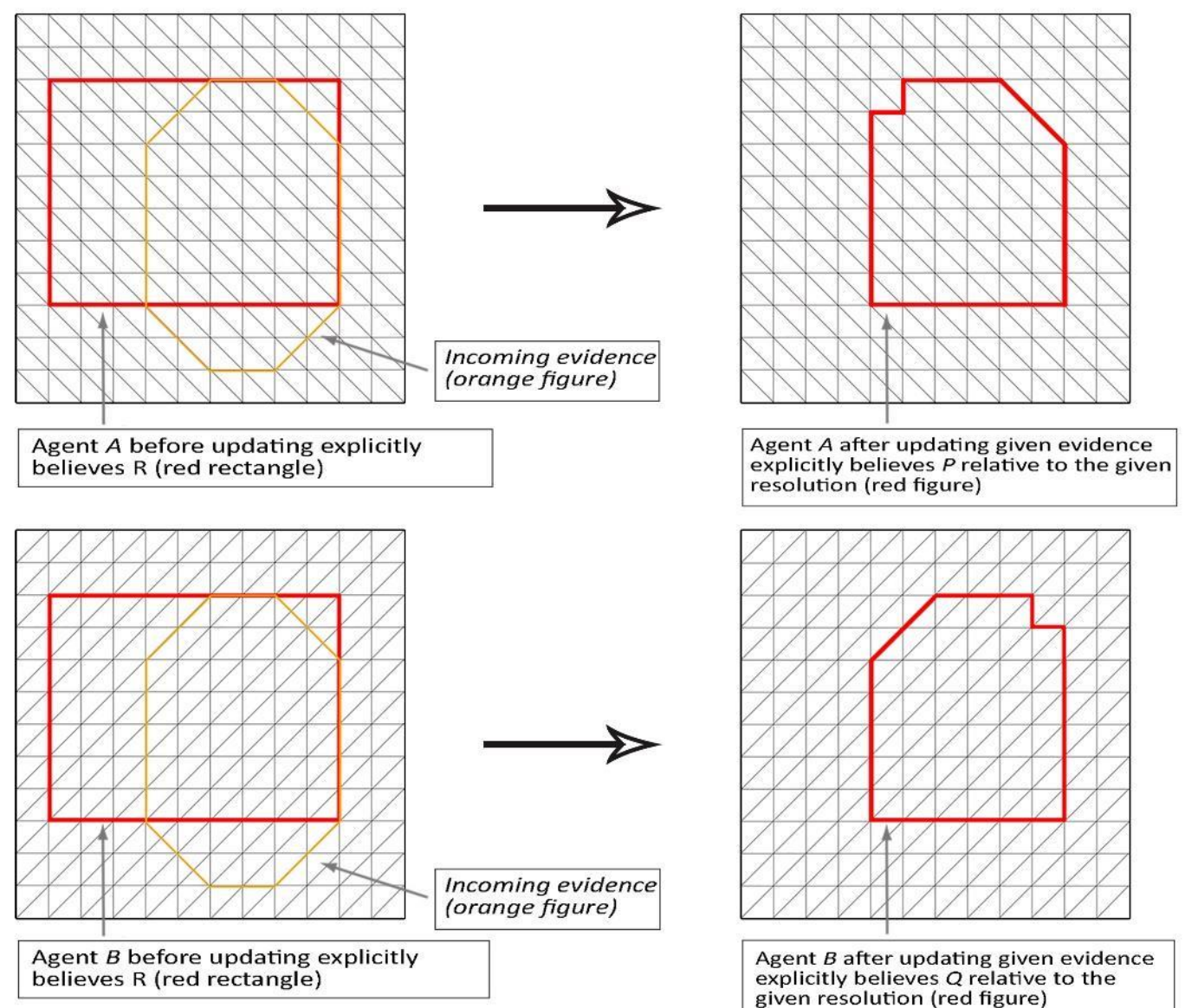
resolution (red figure)

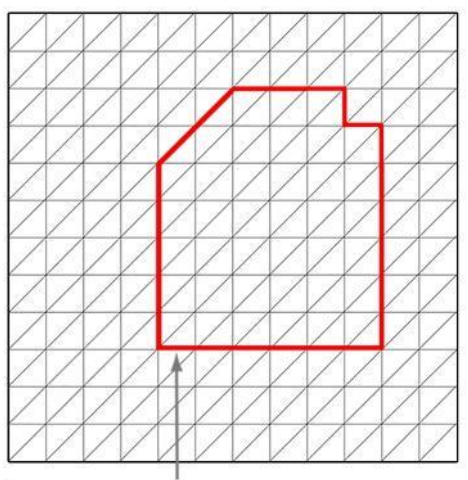

Agent $B$ after updating given evidence explicitly believes $Q$ relative to the given resolution (red figure)

Figure 3: Update of two belief states relative to different resolutions.

On reflection, this result is not surprising. The explicit beliefs of fully rational subjects are not determined by just their evidence; but rather, by their evidence together with the questions to which they are sensitive. ${ }^{7}$ If fully rational subjects can differ with respect to these questions, they can differ in their explicit beliefs.

\subsection{Generalizing to a Quantitative Framework}

Some of the debate about uniqueness is conducted within a quantitative framework, addressing credences rather than outright beliefs and disbeliefs. The reader may wonder whether the considerations presented in the last subsection generalize to a quantitative framework. In this subsection, we show that they do. 
To move to a quantitative framework, we start not with a simple division of modal space into the worlds that are left open and those that are not. Rather, we start with a probability density function over modal space. ${ }^{8}$ Thus, every set of possible worlds is assigned a probability of containing the actual world, and these probabilities obey the Kolmogorov axioms for classical probability. ${ }^{9}$ For each set of worlds, its probability models the implicit credence that a fully rational agent gives to that proposition. We write $\operatorname{ImCr}(P)$ for the implicit credence that a fully rational agent has towards the proposition $P$; i.e., $\operatorname{Im} \operatorname{Cr}(\cdot)$ is our probability density function.

As for our quantitative equivalent of explicit belief, we define the notion of an explicit credence, and we write "ExCr$(P)$ " for the explicit credence that a fully rational agent has towards the proposition $P$ relative to resolution $R$. Resolutions are defined as before, i.e., as partitions of modal space. $\operatorname{ExCr}_{X} Y$ is a function from resolutions and cells of these resolutions to values in the unit interval. Given a resolution, $X$, and cell, $Y$, of that resolution, we define $\operatorname{ExCr}_{X}(Y)$ as $\operatorname{ImCr}(Y)$. It is easy to see that this yields the following: for any $R$, the explicit credence in the trivial proposition, $\operatorname{ExCr}_{R}(\Omega)$, is 1 . All propositions that are unions of cells of $R$ get at least a credence of 0 . And the explicit credence in the union of two disjoint propositions is the sum of the explicit credence in each. Thus, we arrive at a probability function not over all propositions but merely over the algebra formed by the cells of the relevant resolution $R$.

When new evidence, $E$, comes in, we will update the implicit credences of our rational subjects by standard Bayesian conditionalization, i.e., $\operatorname{ImCr}_{\text {post }}(P)=\operatorname{ImCr}_{\text {prior }}(P \mid E)$. Of course, many philosophers have suggested alternative update rules. Here we choose Bayesian conditionalization to illustrate our account. We leave questions like how to construct an analogue of Jeffrey conditionalization for explicit credences for another occasion.

How should rational agents update their explicit credences? Given that the subject has no access to her implicit credences, there must be a way to update explicit credences without appealing to implicit credences. We can do that as follows: First, if the evidence rules out every world in a cell of the agent's resolution, then the agent should set her explicit credence in that cell to zero; i.e., for any $R$, if $E$ entails not- $Y$, then $\operatorname{ExCr}_{R \text { post }}(Y)=0$. Second, we renormalize by 
setting the explicit credence in the union of all the remaining cells to one, i.e., for any $R$, if $U$ is the union of all the cells, $Y$, of $R$ such that $E$ does not entail not- $Y$, then $\operatorname{ExCr}_{R \text { post }}(U)=1$. Now, the ratio of the probability of any given cell to the union, $U$, of the cells that are left open by the evidence should stay constant. ${ }^{10}$ Hence, for any cell $C, \frac{\operatorname{ExCr}_{R \text { prior }}(C)}{\operatorname{ExCr}_{R \text { prior }}(U)}=\frac{E x C r_{R \text { post }}(C)}{E x C r_{R \text { post }}(U)}=$ $\operatorname{ExCr}_{R \text { post }}(C)$. So the agent should set her new explicit credence in cell $C$ of resolution $R$ to the value of the ratio of her prior explicit credence in $C$ relative to $R$ divided by her prior explicit credence in the union of the cells of $R$ that are not ruled out by her new evidence $E$.

This way of updating explicit credences does not always coincide with the result of first updating implicit credences and then deriving what the agent's explicit credences should be from those implicit credences, as we have done above. It is not possible to do that without appealing to the agent's implicit credences. However, the results of the update procedure just described coincide with the procedure that appeals to implicit credence in cases where the evidence cuts exactly along the borders of the resolution at issue. To see this, assume that the boundary of $E$ coincides with the boundary of $Y$. It suffices to show that each cell $C, \operatorname{ImCr}_{\text {prior }}(C \mid E)=$ $\frac{\operatorname{ExCr}_{R \text { prior }}(C)}{\operatorname{ExCr}_{R} \text { prior }(U)}$. Now, $E$ cuts exactly along the borders of $R$. So every cell of $R$ contains either only worlds in which $E$ is true or only worlds in which $E$ is false. But $U$ contains exactly those cells of $R$ in which there is at least one $E$-world. Hence, $E=U$. Moreover, for any cell contained in $U$ the proposition $C$ is equivalent to $C \& U$, which is $C \& E$. Therefore, it suffices to show that $\operatorname{ImCr} r_{\text {prior }}(C \mid E)=\frac{E x C r_{R \text { prior }}(C \& E)}{E x C r_{R \text { prior }}(E)}$. But that is true by the definition of conditional probability.

No matter how we update the explicit credences, we will still get failures of UNIQ. Here, another look at Fig. 3 is helpful. Whether we use Bayesian conditionalization to update implicit credences on our evidence $E$ and then determine explicit credences on that basis or whether we update by using the procedure for explicit credences that we just described, the two subjects in Fig. 3 will assign non-zero probabilities to exactly the cells of their resolutions that contain at least one world in which $E$ is true. These are exactly the cells that are depicted in Fig. 3 as the 
cells that are left open after updating with $E$ in the qualitative framework. And these are also exactly the cells that will receive non-zero explicit credence after updating in the quantitative framework. Since these cells differ for our two subjects, our two subjects end up with different belief states relative to their respective resolutions. Hence, UNIQ fails for explicit credences.

In the previous section, we established that our argument is concerned with permissivism concerning explicit attitudes, and our discussion has mainly focused on beliefs. However, the motivation underlying our argument can be extended towards explicit credences. They, too, can play a key role on guiding how we act or reason. This is obviously so according to theories like rational choice theory, according to which credences are the doxastic attitudes that figure as input into practical reasoning, alongside preferences. It also holds according to theories according to which to believe a proposition is to have a credence in that proposition that is above a certain threshold, i.e., theories that accept the so-called Lockean thesis. At this point, it is worth mentioning that we take Leitgeb's $(2014,2017)$ stability theory of belief, which is one of the most sophisticated theories that underwrite the Lockean thesis, to be congenial to our project. Leitgeb's theory reconciles two principles that are often thought to be incompatible, namely the logical closure of beliefs and the Lockean thesis, i.e., that belief is a credence above a certain threshold. What is interesting is that Leitgeb's theory $(2014,155-156)$ requires that what someone believes is relative to how the space of possibilities is partitioned, i.e., beliefs are relative to resolutions, in our terminology. More precisely, on Leitgeb's theory the threshold that credences must exceed to be beliefs depend on how the worlds are partitioned. Thus, if we accept Leitgeb's stability theory of belief and hold that different partitions (aka resolutions) can be equally rational, we can run a version of our argument above. The same goes for other theories according to which beliefs are relative to resolutions and rationality leaves room for more than one rational resolution. However, two differences between our account and Leitgeb's stability theory are important: First, it is crucial for Leitgeb that rational beliefs are closed under entailment. By contrast, it is important to us that explicit beliefs are not closed under entailment. Secondly, Leitgeb's discussion does not distinguish between implicit and explicit beliefs. We are 
inclined to interpret him as talking about implicit beliefs because his topic are inferentially perfect agents, by which he means that "that the usual logical principles of doxastic closure and the principles of probability can be taken for granted for any such agent which distinguishes a perfectly rational agent that believes all and only true propositions" $(2014$, p. 137). If Leitgeb is right that implicit beliefs are also sensitive to resolutions and, moreover, alternative resolutions can be equally rational, stability theory might open the door to a version of our argument that applies to implicit and not just explicit beliefs. However, we will leave that project for another occasion.

The upshot of this subsection is that our argument for permissivism is not limited to a qualitative way of thinking about doxastic attitudes. An exactly parallel argument applies to credences.

\subsection{Two Rational Subjects Can Differ in Their Resolutions}

In the last two subsections we argued for the first premise of our argument for permissivism, namely that if two fully rational subjects with the same initial doxastic attitudes, the same evidence, and the same epistemic standards can differ in the resolutions for which their explicit beliefs are defined, then UNIQ is false and permissivism is true for explicit beliefs. We now turn to premise (II), which says that two fully rational subjects who meet the conditions just mentioned can indeed differ in the resolutions for which their explicit beliefs are defined. Put differently, we will now argue that two fully rational subjects can be sensitive to different questions. Reformulating again, the mere fact that a fully rational agent with whom you share all evidence etc. is sensitive to a particular question while you are not sensitive to that question does not entail that you are not fully rational.

A difference in the sensitivity to questions can have various causes. While some of these causes may entail that one of the two differing subjects is less than fully rational, there are other cases for which this is not plausible. Consider the following three cases. 
(a) The two subjects are interested in different propositions. In the example with which we started, you are interested in knowing where your keys are while I am interested in knowing where my wallet is.

(b) The two agents use different conceptual frameworks. If, e.g., one of our subjects uses the conceptual framework of the theory of relativity, she will be sensitive to questions like: Are these two events that are simultaneous from my frame of reference also simultaneous from such-and-such other frame of reference? If the other agent uses a Newtonian conceptual framework, she will not be sensitive to such questions.

(c) Due to lack of attention, confusion, motivated reasoning, or the like, one (or both) of our subjects may fail to make some potentially important distinctions between possibilities.

We are happy to grant that the subject in case (c) is not fully rational. It is a failure of rationality to ignore differences between possibilities merely because of confusion or inattention or the like. We submit, however, that this is not true of cases (a) and (b). Regarding (a), being interested in one subject matter rather than another is not a failure of rationality. ${ }^{11}$ And for (b), that you are using one conceptual framework rather than another does not entail that you are less than fully rational. Why might an opponent think otherwise?

An opponent might think that our opinion regarding $(a)$ is the result of running together the rationality of forming certain beliefs in response to evidence and the rationality of gathering certain evidence. The opponent might grant that, in the example, you have excellent reason to seek out evidence regarding the whereabouts of your keys, whereas I have reason to gather evidence about where my wallet is, and not vice versa. But these are practical reasons to gather evidence; they are not theoretical reasons to form different beliefs in response to the same evidence. In response, we want to be clear that our argument does not turn on practical reasons for gathering evidence. Resolutions are not intentions to do anything, let alone intentions to gather evidence. They are essential constituents of explicit belief states. 
To see this, it may help to realize that if two agents share all their practical interests, they could still differ in what they find intellectually stimulating. Suppose, e.g., that our two subjects start with the same implicit beliefs, and that their explicit beliefs rule out exactly the same worlds. They both get evidence that the third of the "Twelve Articles" of the Swabian League from 1525 demanded, by appeal to the bible, the abolishment of serfdom and freedom for all human beings. If one of our subjects has an interest in the history of religion and the other has an interest in the history of emancipatory movements, then they will probably attend to very different consequences of this new piece of information. The first may form the belief that the Swabian League was not impressed with Luther's distinction between the inner and the outer person. The second subject, by contrast, may form the belief that appeals to universal human rights in Europe appeared long before enlightenment philosophers like Locke. Our two subjects were not gathering different evidence. They formed different explicit beliefs in response to the same evidence. They did so because they are sensitive to different questions, which reflects their different interests. We don't see any reason to claim that one of these two subjects is less than fully rational.

The opponent might protest that fully rational agents form beliefs in all of the propositions that are entailed by their evidence. To say that is to say that full rationality requires logical omniscience. We are happy to accept (at least for our purposes here) that implicit beliefs are closed under entailment. We deny, however, that the explicit beliefs of fully rational agents are closed under entailment. We doubt that a conception of full rationality that requires that explicit beliefs are closed under entailment can do any valuable theoretical work. It renders the distinction between implicit and explicit beliefs irrelevant for fully rational agents. But that distinction strikes us as intuitive and theoretically valuable.

Let's now turn to (b). An opponent might hold that cases like (b) involve a failure of rationality because conceptual frameworks are always part of theories and fully rational agents should agree on the theory they accept (if any) about any given subject matter. Hence, fully rational agents who share all their evidence always have the same conceptual frameworks 
available. There are at least three problems with this reply. First, the reply presupposes uniqueness by presupposing that two fully rational agents who share their evidence agree on the theories that they accept. That makes this reply dialectically inadequate. Second, the reply overlooks that even if both agents have the same conceptual framework available, they need not be under any rational obligation to use it in a given situation. After all, we can sometimes describe the same phenomenon within two different theories. In fact, that seems necessary for cases of theory reduction (see also Titelbaum, 2010). Third, the reply implies that if we present our two subjects with new evidence that requires them to invent a new conceptual framework, then it will be a failure of rationality if one of them fails to invent that conceptual framework. That strikes us as implausible. No doubt, we admire inventors of new conceptual frameworks like Aristotle, Newton, Marx, Freud or Anscombe. But it seems inappropriate to think that the contemporaries of these figures who shared their evidence but didn't invent equivalent conceptual frameworks are thereby less than fully rational.

Let's take stock. We have just argued that there can be two fully rational agents who share all of their evidence, prior beliefs, and epistemic standards but who differ with respect to the resolutions over which their explicit beliefs are defined, i.e., with respect to the questions to which they are sensitive. Moreover, we have argued in the previous two subsections that if two fully rational agents can differ in this way, then permissivism is true and uniqueness fails for explicit beliefs, i.e., UNIQ is false. Along the way, we showed that a parallel argument is available for explicit credences. This concludes our argument for permissivism about explicit doxastic attitudes.

\section{Objections}

We want to end by considering some potential worries. We can imagine six ways in which an opponent may try to resist our argument. 
First objection: The argument in this paper requires merely a minor adjustment of UNIQ, namely that we require that the two subjects are sensitive to the same questions. Thus, the advocate of uniqueness should move to UNIQ*:

UNIQ* If two fully rational subjects (1) started with the same initial doxastic attitudes, (2) share all their evidence, (3) use the same epistemic standards, and (4) are sensitive to the same questions, then they must have the same explicit doxastic attitude (or lack thereof) towards any proposition.

In response, we are willing to grant (at least for the purposes of this paper) that UNIQ* is true. However, we don't think that UNIQ* should satisfy any advocate of uniqueness. The initial motivation behind uniqueness, as far as we can see, is to hold that evidential considerations alone determine a unique doxastic state that is fully rational for any given agent. Facts about the agent's interests or values play no role. As we have seen, to which questions an agent is sensitive is, at least in part, a matter of what the agent finds interesting. That is plausibly a matter of the agent's interests and values. Hence, to move to UNIQ* is, in effect, to give up uniqueness.

It is worth pointing out that the fact that we can accept UNIQ* shows that our view is not vulnerable to the so-called "arbitrariness objection." As White $(2005$, p. 448) formulates it, the worry is that, according to permissivism, "whatever I do end up believing upon rational deliberation will depend, if not on blind chance, on some arbitrary factor having no bearing on the matter in question." On our view, what determines what you ought to believe explicitly are the resolutions over which your explicit beliefs are defined. Whether or not the resolutions over which your explicit beliefs are defined are such that you are sensitive to the question whether $P$ has indeed no evidential bearing on whether $P$. Rather, it has a bearing on whether you can form any explicit belief regarding $P$ (without becoming sensitive to new questions). That is not an arbitrary factor with respect to whether you end up believing that $P$.

Second objection: As a follow-up to the worry already addressed in Section $2.3{ }^{12}$ an opponent might hold that an explicit belief is not really a doxastic attitude in any interesting 
sense. Rather, it is an intrapersonal analogue of assertion, and no one would claim that, given a body of evidence, there is a unique set of assertions that an agent is rationally required to make. In response to this worry, we want to stress that our notion of an explicit belief is not an intrapersonal analogue of assertion. Explicit beliefs are beliefs that are (a) not rationally required to be closed under entailment, (b) are integrated with one another only to the extent that they are defined over the same set of questions, and (c) the explicit beliefs that are thus integrated guide our behavior. One may wonder, however, how our explicit beliefs differ from a mere intrapersonal analogue of assertions. The problem comes out when explicit beliefs are defined by the questions one asks and understood as one's stand on these questions. Notice, however, that resolutions are the semantic interpretants of the contents of questions; they do not model the act of asking a question. Explicit beliefs are not inner acts of asserting that answer inner acts of posing questions. Rather our thinking, reasoning and deliberating is sensitive to the contents of certain questions. This can happen because these questions are the contents of some of our interrogative attitudes such as wondering, curiosity, and deliberating (Friedman, 2017). However, we can also be sensitive to a difference between sets of possibilities without engaging in any occurrent or conscious thoughts about this difference.

Third objection: Again related to the issue of Section 2.3 above, ${ }^{13}$ an opponent may worry that all the extant arguments for and against uniqueness are such that they only make sense when we interpret uniqueness as a claim about implicit doxastic attitudes. Hence, our discussion fails to make adequate contact with the literature on uniqueness. In response to this worry, we want to point out that extant arguments can be understood as targeting either implicit or explicit doxastic attitudes. As already intimated in Section 2.3, White (2005; 2013) argues for the importance of being uniquely rational when deliberating practically. Greco \& Hedden (2016) crucially rely on cases of deference, and Dogramaci and Horowitz (2016) rely in their arguments on cases of testimony. These are all cases of explicit beliefs. For the beliefs that occur in practical deliberation or that are passed on via testimony and deference are all explicit beliefs. That is, the agents that hold them are sensitive to the distinctions in modal space that these beliefs draw. 
Otherwise, they couldn't use them in their reasoning and deliberating, and they couldn't express them overtly. Thus, extant arguments for uniqueness are not clearly concerned exclusively with implicit attitudes. If these advocates of uniqueness want to restrict their claims to implicit attitudes, our arguments will no longer apply to them. Since their arguments rely on cases of explicit beliefs, however, it seems to us that the restriction to implicit attitudes will be considered a cost by advocates of uniqueness. Indeed, even if advocates of uniqueness restrict their thesis to implicit attitudes, permissivism about agents' explicit beliefs remains interesting since explicit beliefs are integral to matters such as divergence in opinions, the statements agents assert in testimony and their reliability, how beliefs guide agents through life, and the like.

Fourth objection: An opponent might hold that there are better and worse questions to which an agent can be sensitive, given a body of evidence. A fully rational agent is sensitive to all and only the questions that are good questions, given her evidence. Hence, fully rational agents who share their evidence are always sensitive to the same questions. In response to this objection, we first want to note that this objection implies that there is a unique set of questions to which any fully rational agent with a given body of evidence is sensitive. The objection is a flat-out denial of our premise (II). So, in effect, we already addressed this worry in the previous section. Here we want to add that the objection derives some apparent plausibility from the fact that there are indeed better and worse questions, given a body of evidence. There may indeed be questions such that it is irrational to be sensitive to those questions. Someone who, let's say, cares only about space aliens and is sensitive to few questions that don't concern space aliens may be less than perfectly theoretically rational. Her theoretical vision is, as it were, too limited to count as theoretically rational. We can admit that without undermining our argument. We are not committed to the universal claim that any set of resolutions is equally rational. We are merely committed to the existential claim that there is at least one pair of resolutions such that, given some body of evidence, both resolutions are equally rational. If our opponent has to deny this modest claim, that is a significant cost of her view. 
Fifth objection: An opponent might hold that the notion of explicit beliefs and Yalcin's framework is an attempt to capture a way in which we are less than perfectly rational. For a fully rational agent, the opponent might say, there is no difference between implicit and explicit beliefs. Only less than fully rational subjects who cannot distinguish all possible worlds from each other have any need for explicit beliefs. That worry strikes us as largely terminological. If we want, we can reserve the term "fully rational subject" for a subject who can and does distinguish every possible world from any other possible world in her belief forming processes. But there is surely room for an ideal of rationality that doesn't come with this strong requirement. Yalcin's apparatus gives us a way to conceptualize such an ideal.

Of course, there may be better ways of modeling such an ideal. We suspect, however, that any plausible model will have to include the idea that explicit beliefs are relative to the questions to which the agent is sensitive and, hence, relative to the agent's interests. If that is correct, a version of our argument will go through for any plausible model of the less ambitious ideal of rationality. That being said, we cannot rule out that the advocate of uniqueness finds a way to model the explicit beliefs of fully rational agents who are not logically omniscient that doesn't allow us to run a version of the argument above. Until and unless advocates of uniqueness provide such a model, however, the argument above gives us strong reason to reject uniqueness for explicit beliefs.

Sixth objection: An explicit belief is defined in terms of one's sensitivity to questions thus making the truth of permissivism trivial. The argument for permissivism merely yields the result that one agent fails to explicitly believe something that the other agent believes. The results shown here is too weak to count as a defense of permissivism, for an agent who simply lacks an attitude will ultimately have the same attitude as the other agent if she is made aware of the other agent's set of questions. And a lack of attitude is not the same as positive attitudes, i.e. belief, disbelief, or suspension of judgment. Taking this objection seriously, we provide two considerations. 
First, we consider whether it is plausible for agents to have different positive attitudes. One natural way is to deny closure. What the agents believe are their explicit beliefs and nothing more. Having different questions will then result in having different attitudes. However, since we grant uniqueness for implicit beliefs, we can rule out this option. A more substantial response is to say that making implicit beliefs explicit takes more than just raising the questions one needs to explicitly believe an implicit belief. ${ }^{14}$ An agent may have to retrieve from memory all that she explicitly believes and make the necessary inferences from those beliefs. In some cases, this can be easily done. But in other cases, this will presuppose that an agent has infinite computational power. We believe our discussion of agents being slightly short of an ideal of rationality suffices. If that is correct, then we can grant that our agents do not have infinite computational power. And if this holds, it is possible for our agents to have different positive attitudes.

Second, we consider that having different positive attitudes is a non-option. Our agents fail to have an attitude towards the same propositions since they do not hold the same resolutions. Our argument for permissivism remains interesting as there are consequences for one's attitudes and non-attitudes. We might testify or act differently if we do not hold the same beliefs. Consider our history example above. When agents hold different explicit beliefs, they will differ on what they say about the significance of the third of the "Twelve Articles."

\section{Conclusion}

Let's sum up. We have provided a new argument for permissivism. Our argument supports a moderate, interpersonal version of permissivism about explicit beliefs. The core idea is that two fully rational subjects who share all their evidence, prior beliefs, and epistemic standards may nevertheless differ in the questions to which their explicit beliefs are sensitive. This may happen, e.g., if they have different theoretical interests or if they use different conceptual frameworks. Such differences don't imply that one of the subjects is less than fully rational. We have shown, 
however, that these differences can yield different explicit doxastic attitudes towards the same proposition. Hence, uniqueness fails.

Our version of permissivism is modest in several respects. It applies only to explicit doxastic attitudes. And it doesn't allow that two fully rational agents with the same evidence are such that one explicitly believes a certain proposition and the other explicitly disbelieves that proposition. On the other hand, our version of permissivism doesn't rely on rationally permissible differences in epistemic standards or ur-priors or the like.

Let us end by pointing out a larger question that our argument raises. We say that the explicit beliefs of fully rational agents are not uniquely determined by their evidence but only by their evidence together with their interests (or the questions to which they are sensitive). We have admitted that it may sometimes constitute a failure of rationality to have certain interests or to be sensitive to certain questions. Is that irrationality of the practical or of the theoretical variety? Neither option strikes us as obviously correct. The question deserves our attention. But it will have to wait for another occasion. ${ }^{15}$

${ }^{1}$ We take it that not having any explicit attitude towards a certain proposition counts as a different attitude (cf. Friedman, 2018).

2 Here we ignore, of course, de se beliefs.

${ }^{3}$ We follow Yalcin in taking these worlds to be the metaphysically possible worlds. Since the problem of logical omniscience that drives Yalcin arises even for merely logically possible worlds, moving to epistemically possible worlds will be an alternative only if some epistemically possible worlds are logically impossible.

4 The same is true, of course, of metaphysical entailment if we don't include any metaphysically impossible worlds, and similarly for other kinds of modality.

${ }^{5}$ This last cell is already ruled out by an implicature of the question. But we ignore such complications here.

${ }^{6}$ We would like to thank an anonymous referee for pressing us on this point.

${ }^{7}$ The idea is not entirely new. We would like to thank an anonymous referee for pointing this out. Nelson (2010), for example, argues that we do not have positive epistemic duties such as 'you ought to believe $\varphi^{\prime}$. Our evidence only limits what is permissible to believe. Such positive duties are instead determined by non-epistemic considerations which include one's needs, interests, and preferences. In this case, the relevant non-epistemic consideration are the questions to which they are sensitive. However, whether questions are non-epistemic considerations is currently up for debate (see, e.g., Friedman, forthcoming).

8 We have to take probability density function and not simply a probability function because there are infinitely, indeed uncountably, many possible worlds.

${ }^{9}$ That is, the probability of the set of all possible worlds is 1 ; all sets of worlds have a probability of at least 0 ; and the probability of the union of disjoint sets of possible worlds is the sum of the probability of each.

10 At least, that is the principle behind Bayesian conditionalization, of which we want to develop an analogue for explicit credences. The constancy of these ratios may be the source of problems with undercutting defeat (Weisberg, 2015). 


\footnotetext{
${ }^{11}$ Jane Friedman (2018) has argued that we are not permitted to believe propositions that are not of interest to us. If that is true, we could strengthen our conclusion to the claim that two agents with the same evidence and prior beliefs are sometimes not permitted to believe the same proposition. However, here we stay neutral regarding this point.

${ }^{12}$ Thanks to an anonymous referee for pressing us on this point.

${ }^{13}$ Thanks to an anonymous referee for pressing us on this point.

${ }^{14}$ See Velázquez-Quesada (2014) for a discussion on how certain acts of inference are used to derive explicit from implicit beliefs.

${ }^{15}$ For helpful feedback and comments on earlier versions of the paper, we would like to thank Matthew Barker, Matthew Burley, George Christopoulos, Zeno Şerban, Jordan Walters, Ali Aenehzodae, Joshua Brecka, participants of the conferences by the Ohio Philosophical Association and the Canadian Philosophical Association, and two anonymous referees. We would also like to thank Densy Jimenez for his help with the figures.
}

\section{References}

Christensen, D. (2007). Epistemology of disagreement: The good news. Philosophical Review, 116(2), 187217.

Dogramaci, S., \& Horowitz, S. (2016). An Argument for Uniqueness About Evidential Support. Philosophical Issues, 26(1), 130-147.

Elga, A. (2007). Reflection and disagreement. Noûs, 41(3), 478-502.

Feldman, R. (2007). Reasonable religious disagreements. In L. Antony (Ed.), Philosophers Without Gods: Meditations on Atheism and the Secular Life (pp. 194-214). Oxford: Oxford University Press.

Friedman, J. (2017). Why Suspend Judging?. Noûs, 51(2), 302-326.

Friedman, J. (2018). Junk Beliefs and Interest-Driven Epistemology. Philosophy and Phenomenological Research, 97(3), 568-583.

Friedman, J. (forthcoming). The Epistemic and The Zetetic. Philosophical Review.

Greco, D., \& Hedden, B. (2016). Uniqueness and Metaepistemology. Journal of Philosophy, 113(8), 365395.

Hamblin, C. L. (1958). Questions. Australasian Journal of Philosophy, 36(3), 159 - 168.

Horowitz, S. (2014). Immoderately rational. Philosophical Studies, 167(1), 41-56.

Horowitz, S. (2018). Epistemic Value and the Jamesian Goals. In J. D. Kristoffer Ahlstrom-Vij (Ed.), Epistemic Consequentialism (pp. forthcoming). Oxford: Oxford University Press.

Horowitz, S. (2019). The Truth Problem for Permissivism. Journal of Philosophy, 116(5), 237-262.

Jackson, E. (2019). A Defense of Intrapersonal Belief Permissivism. Episteme, 1-15. doi:10.1017/epi.2019.19

Kelly, T. (2013). Evidence Can Be Permissive. In M. Steup \& J. Turri (Eds.), Contemporary Debates in Epistemology (pp. 298-311). Hoboken: Wiley \& Sons.

Kopec, M., and Titelbaum, M. G. (2016). The Uniqueness Thesis. Philosophy Compass, 11: 189- 200.

Leitgeb, H. (2014). The Stability Theory of Belief. The Philosophical Review, 123(2), 131-171.

Leitgeb, H. (2017). The Stability of Belief: How Rational Belief Coheres with Probability. Oxford University Press. 
Levinstein, B. A. (2017). Permissive Rationality and Sensitivity. Philosophy and Phenomenological Research, 94(2), 342-370.

Matheson, J. (2011). The Case for Rational Uniqueness. Logic and Episteme, 2(3), 359-373.

Meacham, C. J. G. (2013). Impermissive Bayesianism. Erkenntnis(S6), 1-33.

Meacham, C. J. G. (2019). Deference and Uniqueness. Philosophical Studies, 176(3), 709-732.

Nelson, M. T. (2010). We Have No Positive Epistemic Duties. Mind, 119(473), 83-102.

Raleigh, T. (2017). Another Argument Against Uniqueness. Philosophical Quarterly, 67(267), 327-346.

Rosa, L. (2012). Justification and the Uniqueness Thesis. Logos and Episteme(4), 571-577.

Rosen, G. (2001). Nominalism, Naturalism, Epistemic Relativism. Noûs, 35(s15), 69 - 91.

Schoenfield, M. (2014). Permission to Believe: Why Permissivism Is True and What It Tells Us About Irrelevant Influences on Belief. Noûs, 48(2), 193-218.

Schoenfield, M. (2018). Permissivism and the Value of Rationality A Challenge to the Uniqueness Thesis. Philosophy and Phenomenological Research, online first. doi:10.1111/phpr.12490

Schultheis, G. (2018). Living on the Edge: Against Epistemic Permissivism. Mind, 127(507), 863-879.

Simpson, R. M. (2017). Permissivism and The Arbitrariness Objection. Episteme, 14(4), 519-538.

Smithies, D. (2015). Ideal Rationality and Logical Omniscience. Synthese, 192(9), 2769-2793.

Stalnaker, R. (1984). Inquiry. Cambridge: Cambridge University Press.

Stalnaker, R. (1991). The problem of logical omniscience, I. Synthese, 89(3), 425 - 440.

Stapleford, S. (2018). Intraspecies Impermissivism. Episteme, 1-17.

Thorstad, D. (2019). Permissive Metaepistemology. Mind, 128(511), 907-926.

Titelbaum, M. G. (2010). Not enough there there evidence, reasons, and language independence. Philosophical Perspectives, 24(1), 477-528.

Titelbaum, M. G., \& Kopec, M. (2019). When Rational Reasoners Reason Differently. In M. BalcerakJackson; \& B. Balcerak-Jackson (Eds.), Reasoning: New Essays on Theoretical and Practical Thinking (pp. 205-231). Oxford: Oxford University Press.

Velázquez-Quesada, F. R. (2014). Dynamic epistemic logic for implicit and explicit beliefs. Journal of Logic, Language and Information, 23(2), 107-140.

Weisberg, J. (2015). Updating, Undermining, and Independence. British Journal for the Philosophy of Science, 66(1), 121-159.

White, R. (2005). Epistemic permissiveness. Philosophical Perspectives, 19(1), 445-459.

White, R. (2013). Evidence Cannot Be Permissive. In M. Steup \& J. Turri (Eds.), Contemporary Debates in Epistemology (pp. 312): Blackwell.

Yalcin, S. (2018). Belief as Question-Sensitive. Philosophy and Phenomenological Research, 97(1), 23-47. 